\title{
RUNWAY INCURSION REDUCTION PROGRAM DALLAS/FT.WORTH DEMONSTRATION/VALIDATION PROJECT
}

\author{
Arthur Sullivan, Federal Aviation Administration, Washington, D.C. \\ Michael Curry, Trios Associates, Inc., Lanham, MD
}

\section{Background}

Runway Incursions (RI's) in the United States have experienced a $16 \%$ average yearly increase from 1993 to $1997 .^{1}$ This fact, coupled with recent airport surface accidents in Atlanta, Detroit, Los Angeles and St. Louis, ranks RI's as a top priority in the Federal Aviation Administration (FAA) and is listed on the National Transportation Safety Board's (NTSB) "10 Most Wanted" safety improvements. ${ }^{2}$

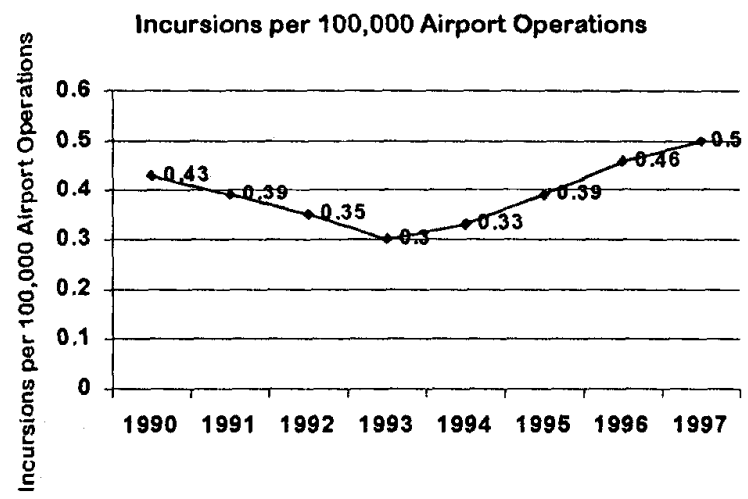

Figure 1- Rates of Runway Incursions, 1990 Through $1997^{3}$

It is generally accepted that increased situational awareness among all users and improved surveillance of the airport will be effective in reducing RI's that could lead to accidents. To this end, seamless surveillance coverage, identification of aircraft on the surface and information sharing with airport

\footnotetext{
1 "1997 Aviation Safety Statistical Handbook," Office of System Safety, FAA; 1998.

2 "Most Wanted Transportation Safety Improvements," NTSB; May 5, 1998.

${ }^{3}$ Ibid.
}

\section{U.S. Government work not protected by U.S. Copyright.}

users have become a priority for the FAA. This priority is reflected in the FAA's "Concept of Operations for the National Airspace System (NAS) in 2005,"4 the NAS Architecture, ${ }^{5}$ and the 1998 Surface Operations Action Plan.

The Runway Incursion Reduction Program (RIRP) is working in coordination with industry and other Government agencies in research and evaluation of nondevelopmental items (NDI) and commercial off-the-shelf (COTS) technologies in an effort to aid in the reduction of runway incursions. The goals of this effort will be to address the following areas of need:

- Seamless Coverage - The ability to accurately determine the position of aircraft and vehicles with no gap in coverage while on the airport surface.

- Shared Situational Awareness - The ability to provide situational awareness to pilots and vehicle operators as well as controllers.

- Conflict Alerting Capabilities - The ability to provide conflict alerting capabilities for controllers and vehicle operators.

The RIRP will conduct test and demonstration activities to validate the concepts and solutions developed to attain these goals. Dallas-Ft. Worth International Airport (DFW) has been chosen to host these activities.

\footnotetext{
${ }^{4}$ Air Traffic Services Concept of Operations; FAA; April 1997.

${ }^{5}$ NAS Architecture, V. 3.0, DRAFT; FAA; December 1997.

6 "1998 Airport Surface Operations Safety Plan to Prevent Runway Incursions and Improve Surface Operations," Runway Incursion Program Office (ATO-102), FAA; 1998.
} 


\section{Previous Validation Activities - Atlanta Hartsfield Demonstration}

The concepts and systems being validated in DFW have been developed during previous RIRP activities. A joint National Aeronautics and Space Administration (NASA) I FAA demonstration was conducted at Atlanta's Hartsfield International Airport (ATL) during the summer of 1997. This demonstration utilized earlier prototypes of many of the systems being demonstrated at DFW. For more information on the Atlanta Demonstration, please see the publication entitled "Runway Incursion Reduction Program Surveillance System, NASA/FAA Atlanta Demonstration."7

\section{DFW Background}

Dallas-Ft. Worth International Airport is a Category 5 airport (highest traffic level) located 12 miles northwest of Dallas, TX. It ranks first in the United States in aircraft operations, and fourth in enplanements. ${ }^{8}$ However, the FAA's Office of System Capacity projects that, by 2011 , operations and enplanements will grow at rates of $55 \%$ and $78 \%$ respectively, to make DFW the busiest and most highly used airport in the nation.

Currently, DFW has seven runways in both parallel and crossing configurations. DFW maintains most major surveillance and automation systems available in the NAS. The automation system for DFW's Terminal Radar Control (TRACON) is an Automated Radar Terminal System - Model IIIE (ARTS-IIIE). Terminal surveillance is provided by two Airport Surveillance Radar - Model 9 (ASR-9) radars collocated with two Mode Select (Mode S) radars operating in Interim Beacon Interrogator (IBI) mode. Surface surveillance

\footnotetext{
7 "Runway Incursion Reduction Program Surveillance System, NASA/FAA Atlanta Demonstration," V. Capezzuto, M. Curry, S. Pendergast, D. Olster; $17^{\text {th }}$ DASC, 1998.

8 "1998 Aviation Capacity Enhancement (ACE) Plan," FAA

Office of System Capacity; December 1998; pp. 58, A-2, A-5 and A-8.
}

U.S. Government work not protected by U.S. Copyright. is currently provided by two Airport Surface Detection Equipment - Model 3. (ASDE-3) radars, located on the east Air Traffic Control Tower (ATCT) and one on the center ATCT (a third ATCT exists on the west side of the airport).

\section{RIRP DFW Surveillance System}

Figure 2 at the end of this paper shows a block diagram of the RIRP DFW surveillance system. At the heart of the system is the Surface Surveillance Data Server (SSDS). The SSDS receives surveillance and flight plan information from a variety of sources and "fuses" the information into one optimal report for user display. The surveillance information comes from a variety of sensors, including terminal and surface radar, loop technology, Automatic Dependent Surveillance - Broadcast (ADS-B), and multilateration. The flight plan information is received from the local automation system, an ARTS-IIIE. The majority of information is passed between subsystems over a Local Area Network (LAN). It is envisioned that the system will be modular to meet the surveillance requirements of various airports in the U.S.

\section{Surface Surveillance Data Server}

While enabling the SSDS to perform its fusion and safety logic features, the SSDS is being developed on COTS equipment. The SSDS consists of a SPARC workstation in a VME chassis. It also contains scan converters to display ASDE-3 video, and provides the LAN backbone for the RIRP DFW system. The SSDS is being developed by the Volpe National Transportation Systems Center.

\section{Sensor Inputs}

The RIRP DFW demonstration system will accept various surveillance inputs to demonstrate a robust configuration capable of being adapted to any airport. The paragraphs below detail the systems providing surveillance to the SSDS. 


\section{ATIDS}

The Airport Target Identification System (ATIDS) is a combined ADS-B and multilateration system designed to track aircraft in the coverage volume of the airport surface. ${ }^{9}$ Mode $S$ transponder equipped aircraft are tracked using the "squitters" periodically transmitted from the aircraft. ATCRBS transponder equipped aircraft are tracked utilizing a "whisper-shout" sequence to elicit transmissions from the aircraft. These transmissions from both the Mode $\mathrm{S}$ and ATCRBS equipped aircraft are then received by six receiver/transmitters located around the east surface of DFW. Time difference of arrival (TDOA) calculations are performed on the receptions to produce a position report.

Additionally, ATIDS can receive ADS-B transmissions on the $1090 \mathrm{MHz}$ frequency to attain position information. The position report generated through one of the above techniques, and additional information contained in the aircraft transmissions, is passed on to the SSDS for inclusion in a fusion solution via the RIRP DFW system LAN. The ATIDS system is being leased from the Sensis Corporation.

\section{Vehicle ADS-B}

The Vehicle ADS-B system utilizes ADS-B concepts to provide surveillance on airport surface vehicles, such as emergency equipment, to controllers as well as to provide situational awareness to vehicle operators. Each equipped vehicle determines its own position utilizing differential Global Positioning System (DGPS) technology and broadcasts the vehicle position and its identification to three base stations covering the airport. This position and identification information is gathered by a Master Base Station that interfaces with the RIRP LAN via the Data Link Manager to provide the SSDS with the information.

\footnotetext{
9 'The coverage volume includes the complete surface movement area (taxiways and runways), two mile arrival corridors and $300 \mathrm{ft}$. above the surface.
} not protected by U.S. Copyright.
The system also has the capability to provide situational awareness of aircraft and other targets from the SSDS, as well as runway hold bar information. The vehicle ADS-B system was originally developed by Sensis Corporation for the FAA and has been subsequently modified by Trios Associates, Inc.

\section{Data Link Manager}

The Data Link Manager (DLM) serves as a gateway between the RIRP LAN and external systems. These systems include the Vehicle ADS-B system, the LAAS ground station, and future data link communication projects. The DLM provides an interface for target reports to be exchanged between the Vehicle ADS-B system and the RIRP LAN, as well as providing the safety alerts from the RIRP system to be uplinked to vehicle operators and pilots. The DLM is being developed by Trios Associates, Inc.

\section{Loop Technology}

The RIRP system also utilizes Loop Technology to aid in the reduction of gaps in coverage. Inductive loops, similar to those used in automotive traffic systems (e.g., traffic light triggering), are placed in a runway or taxiway that lacks surveillance. An aircraft or vehicle passing over a loop produces an electromagnetic signature that can be processed into a position report. Loop technology will serve the DFW RIRP system by eliminating a coverage gap caused by an airline hangar in the middle of the east side of DFW. It will connect to the SSDS via the RIRP DFW system LAN.

\section{ASDE-3}

The ASDE-3 is a certified, production primary radar intended for airport surface. It maintains a one second update rate and will provide the RIRP DFW system with raw digitized video.

\section{ASR-9}

The ASR-9 is also a certified, production terminal radar system that covers a $60 \mathrm{nmi}$ range. The ASR-9 maintains an approximate five second update rate. The 
RIRP DFW system will utilize position data on arrival aircraft from the ASR-9.

\section{Additional Inputs}

\section{LAAS}

The RIRP DFW system will utilize a LAAS ground station to provide DGPS corrections. These corrections will be available on the LAN through the DLM for various systems to uplink them to aircraft and vehicles.

\section{ARTS-IIIE}

The RIRP DFW system will also utilize an input from the ARTS-IIIE automation system to provide flight plan information for arrival and departure aircraft.

\section{Outputs}

\section{Controller Displays}

The SSDS will provide a color display for the air traffic controllers. For each aircraft, the display will show an optimal position report and identification. Additionally, the display will show incursion alerts and hold bar information.

\section{Aircraft Displays}

The RIRP DFW system will also have the capability to uplink traffic and safety alerts from the SSDS to developmental displays. These displays will be placed in the rear of two test aircraft, in an observation/lab area. These aircraft will be provided by the FAA's William J. Hughes Technical Center. The information will be uplinked via the Data Link Manager utilizing the Vehicle ADS-B system.

\section{Vehicle Displays}

As previously mentioned, the Vehicle ADS-B system will provide position, identification and safety alert information to vehicle operators. A test van equipped with Vehicle ADS-B and other equipment (such as an aircraft transponder) will be utilized during the tests. The test vehicle is being provided by Trios Associates, Inc.

\section{Demonstration/Validation Program}

The following paragraphs summarize the overall plan for demonstrating and validating the RIRP DFW system.

\section{Subsystem Evaluations/Integration Tests}

Each developmental subsystem will be tested and evaluated individually before integration of the entire system. These tests will measure subsystem performance and will also serve to characterize the inputs into the SSDS. Information from these tests will be useful in determining theoretical performance of the entire system. Test plans and reports will be generated for each of the subsystem tests undertaken.

The subsystems will be integrated in steps to isolate problems. As part of the integration process, testing will be done to ensure proper operation between all systems.

\section{RIRP DFW System Tests}

After the subsystem evaluation and integration tests are complete, an entire end-toend system evaluation will occur. These tests will measure performance parameters such as latency, coverage, etc. The test plan for this activity is in development.

\section{Demonstration}

Once a complete system integration and evaluation has been completed, the RIRP DFW system will be presented to interested parties. The purpose of this demonstration will be to obtain comments on the capabilities that the system affords end users. It is expected that the audience for this demonstration will include air traffic controllers, industry representatives, FAA executives and local and regional personnel. 


\section{Future Efforts}

The RIRP program intends to build upon the experience that will be gained during this demonstration/validation project, with the end goal of a certifiable production system that is beneficial to the NAS. Possible future efforts include a second joint FAA/NASA demonstration, leveraging the combined capabilities of the two organizations.

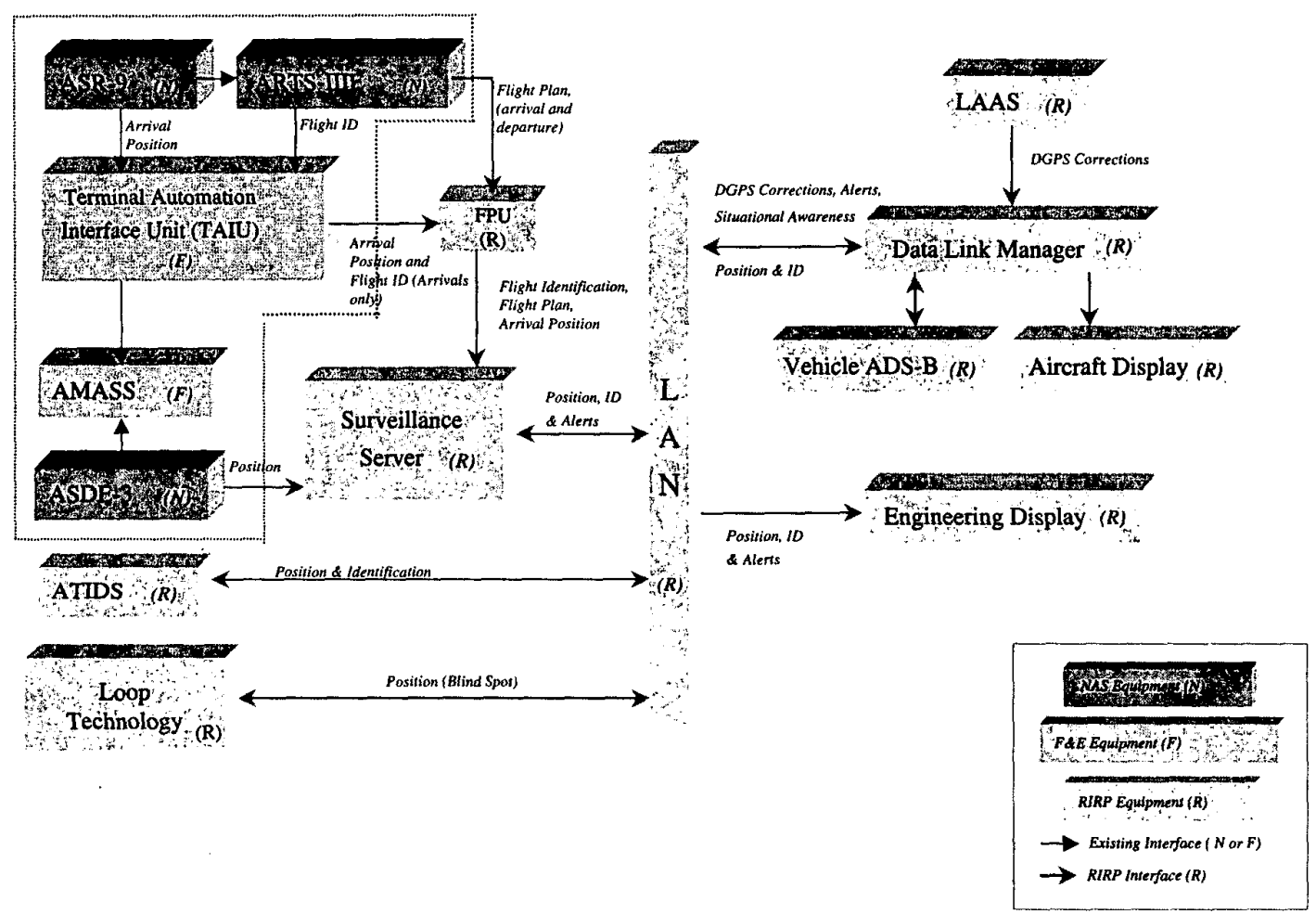

Figure 2: RIRP DFW System Block Diagram 